\title{
Aportes para la construcción de "Universidad Ciudad del conocimiento". La experiencia del Campus Virtual de la Universidad Nacional de Lanús
}

\author{
Contributions for the construction of the "University City of \\ knowledge". The experience of the Virtual Campus of the National \\ University of Lanus
}

\author{
Laura Virginia Garbarini ${ }^{1}$ y María Sol Quiroga ${ }^{2}$
}

\begin{abstract}
${ }^{1}$ Dirección del Campus Virtual, Vicerrectorado, Universidad Nacional de Lanús, Lanús, Argentina.
${ }^{2}$ Departamento de Desarrollo Productivo y Tecnológico, Universidad Nacional de Lanús, Lanús, Argentina. lgarbarini@unla.edu.ar, mquiroga@unla.edu.ar
\end{abstract}

(Recibido: 15 noviembre 2019; aceptado: 03 febrero 2020)

\begin{abstract}
Resumen. La Universidad Nacional de Lanús es una universidad pública y autónoma de la República Argentina, creada en 1995, y que se caracteriza por estar establecida en la Provincia de Buenos Aires. Se considera a sí misma como urbana y comprometida con la resolución de los problemas regionales y nacionales. El proyecto institucional acá presentado promueve la construcción de una ciudad del conocimiento que supere la idea de universidad claustro y articule localmente a los productores de saberes científicos, socioculturales, tecnológicos y políticos. Desde la Dirección del Campus virtual se ha planteado un modelo pedagógico cuyas propuestas educativas estén basadas en los principios rectores de la actividad académica de la UNLa: transdisciplinariedad, problematización de los saberes y modalidad de trabajo en taller. En el presente texto se comparte el proyecto con la creencia de que este ha fortalecido la propuesta educativa de calidad en la institución, la democratización de la enseñanza y la inclusión social.
\end{abstract}

Palabra clave: Universidad, Conocimiento, Campus virtual, Proyecto pedagógico, Entornos virtuales.

Abstract. The National University of Lanús is a public and autonomous university of Argentine Republic, created in 1995, and characterized by being established in the Province of Buenos Aires and considers itself as urban and committed to the resolution of regional and national problems. The institutional project presented in this document promotes the construction of a city of knowledge that exceeds the idea of university cloister and articulates locally producers of scientific, sociocultural, technological and political knowledge. From the Virtual Campus Management, a pedagogical model has been proposed whose educational proposals are based on the guiding principles of the academic activity of the UNLa: transdisciplinarity, problematization of knowledge and modality of workshop work. This article presents the project in the belief that it has strengthened the quality educational proposal in the institution, the democratization of education and social inclusion.

Keywords: University, Knowledge, Virtual campus, Pedagogical project, Virtual environments. 


\section{Introducción}

La Universidad Nacional de Lanús es una institución de educación superior pública y autónoma de la República Argentina. Creada en 1995, se caracteriza por estar establecida en el conurbano de la Provincia de Buenos Aires, zona suburbanizada a partir de la industrialización por sustitución de importaciones, y cuyo crecimiento en la década de 1930 le otorgó un perfil particular. Es un escenario de radicación de migrantes internos sobre una población de inmigrantes ya asentada y presenta una alta densidad de población por estar ubicada cerca del acceso a la Ciudad Autónoma de Buenos Aires. También se caracteriza por su alta densidad institucional, compuesta por gran cantidad de organizaciones civiles, culturales, educativas, recreativas y pequeñas y medianas empresas, gracias a las cuales se genera un entramado social que enriquece las posibilidades de vinculación y articulación con la comunidad.

Así, desde sus inicios la UNLa se ha considerado a sí misma como una universidad urbana, comprometida con el medio local y con la resolución de los problemas regionales y nacionales. En este trabajo se desarrolla el contexto institucional en el que se inscriben las misiones, funciones y tareas del Campus virtual UNLa, su desarrollo y el modelo pedagógico que lo sustenta. Finalmente se harán algunas reflexiones a modo de conclusión sobre los desafíos pendientes.

\section{Contexto institucional}

Según el artículo n. ${ }^{\circ} 2$ de su estatuto, la Universidad Nacional de Lanús "tiene como misión primaria contribuir a través de la producción y distribución de conocimiento y de innovaciones científico tecnológicas, al desarrollo económico, social y cultural de la región, a fin de mejorar su calidad de vida y fortalecer los valores democráticos en el conjunto de la sociedad, articulando el conocimiento universal con los saberes producidos por su comunidad" (Universidad Nacional de Lanús, 2014, p. 2).

Fiel a este principio, la UNLa, que se define a sí misma como una universidad urbana y comprometida con la comunidad, organiza su estructura académica en torno a problemas sociales y no a disciplinas, con el fin de generar conocimientos que, a la hora de intervenir sobre la realidad, deriven con eficacia en la toma de decisiones. Es decir, la UNLa se propone crear conocimiento por medio de la investigación, a partir de problemas regionales y, particularmente, de los que son parte de su entorno.

En el marco de la misión social de la universidad que prioriza la democratización de la enseñanza, se establece el desarrollo de una propuesta educativa basada en tres ejes fundamentales relacionados entre sí:

La articulación de saberes, que propone como estrategia educativa la transdisciplinariedad, reforzando los vínculos de enseñanza entre las diferentes carreras y áreas problemáticas de la UNLa.

La Problematización de saberes, que es definición institucional de que organizar los saberes en torno a problemáticas se vincula con el reto de la alfabetización digital, que supone un desafío a la hora de sistematizar la información y de generar nuevos conocimientos; por último,

El tercer eje se relaciona con la modalidad de trabajo en taller, que organiza la producción del conocimiento en un encuadre áulico, tanto presencial como virtual, de circulación de los saberes y de reconstrucción del conocimiento, y que permite la relación entre teoría y práctica en el aprendizaje simulando escenarios de situaciones profesionales.

\section{Marco teórico del modelo pedagógico}

En la actual sociedad del conocimiento, la integración de las TIC en los procesos de enseñanza y aprendizaje influyeron en la transformación de la idea de educación a distancia.

Siguiendo a Fainholc (1999, p. 24), la educación a distancia puede definirse a partir de dos elementos principales: la separación física del profesor y el estudiante, y el control volitivo que el estudiante asume del proceso de aprendizaje. Desde sus comienzos en la era industrial moderna, las propuestas de la educación a distancia utilizaron los medios tecnológicos disponibles para generar las mismas condiciones que se daban en el aprendizaje presencial (envío de materiales por correspondencia, la radio y más adelante la televisión).

Con el desarrollo de Internet, un primer momento estuvo marcado por el uso del correo electrónico, lo que permitía una llegada más rápida y eficaz de los materiales de estudio, aunque el modelo pedagógico tradicional no se vio sustancialmente modificado (clases expositivas donde el docente era considerado el único depositario del saber). Más adelante, el advenimiento de las plataformas de aprendizaje marcó un verdadero cambio en la concepción de la educación a distancia, ya que estas ofrecen la posibilidad de 
publicar materiales de distinto tipo y en diversos formatos, además de crear espacios de comunicación e interacción entre los estudiantes y el docente.

E-learning y educación virtual fueron conceptos que se utilizaron para denominar estas nuevas formas de acercarse al conocimiento y promover procesos de aprendizaje a distancia.

En este contexto, son los desarrollos de la denominada Web 2.0 los que abren una nueva etapa transformando completamente el modelo de la educación a distancia hasta el punto de no concebir el uso de las tecnologías con el fin de "acortar la distancia", propio del modelo original de la educación a distancia, sino en proponer un nuevo escenario que permita el desarrollo de los procesos de construcción del conocimiento a través de la interacción entre pares con las fuentes de información. Cabe resaltar que la diferencia entre la Web 1.0 y la 2.0 radica en el hecho de que la última es interactiva y se fundamenta en la participación, la colaboración, la producción conjunta y compartida de conocimientos; impregnando las propuestas educativas.

García Aretio et al., (2007) clarifican la idea de "educación a distancia" desarrollada en el contexto de Internet. Argumentan que la educación a distancia no excluye al aula tradicional; de hecho, ambas modalidades poseen lógicas similares: los canales de comunicación y los recursos en los que se ha apoyado la educación a distancia desde sus inicios dieron por supuesta la separación espacio-temporal, por lo que la interacción entre los agentes principales era sumamente limitada. De ahí que las metodologías no presenciales fueran consideradas por muchos una enseñanza de segunda clase. La separación física no implica necesariamente una separación funcional cuando el marco de acción se traslada al ciberespacio. Así, la educación virtual puede darse en cualquier lugar y momento sin recintos precisos ni cerrados, sin que los aspectos temporales y geográficos sean un obstáculo. Por lo tanto, hoy las tecnologías digitales crean nuevos espacios para la interacción pedagógica, permitiendo trascender el papel de auxiliar didáctico que usualmente se le asignaba a la tecnología educativa. Dejan de ser solo un medio o recurso didáctico para convertirse en un espacio material, simbólico y social en el que se desenvuelven las acciones educativas con una impronta participativa y constructivista.

Autores como el sociólogo Jeremy Rifkin advierten que habría que centrar la atención en las nuevas prácticas culturales y mentalidades que han madurado alrededor de las nuevas herramientas y plataformas tecnológicas, como puede observarse en la Web 2.0, con un papel más activo del usuario, que se transforma de consumidor en prosumidor (Rifkin, 2014, p. 246).

Con relación a estos cambios complejos en la educación, Julio Cabero Almenara (2015) dice que la incorporación de la tecnología a la educación puede pensarse desde tres posiciones que el autor denomina “TIC, TAC y TEP" (p. 22).

Desde la perspectiva de las TIC (Tecnologías de la Información y Comunicación), los recursos tecnológicos son percibidos como facilitadores para la transmisión de información y adaptables a cualquier situación de enseñanza, independientes de las características de los estudiantes, lo que permite conseguir con ellos una verdadera formación audiovisual, multimedia e hipertextual. Desde esta posición, los conocimientos que el docente debe tener para su utilización se centran en la vertiente tecnológica e instrumental, destacándose su visión transmisora.

Desde la posición de las TAC (Tecnologías para el Aprendizaje y el Conocimiento), se piensa la tecnología como un instrumento facilitador del aprendizaje, con el objetivo de aprender de manera significativa. Lo importante en esta visión es qué estrategias y metodologías se aplican para alcanzar los objetivos previstos y crear nuevos escenarios de comunicación para el aprendizaje, reflexionando cómo puede usarse la tecnología para modificar la práctica docente.

Desde la posición de las TEP (Tecnologías para el Empoderamiento y la Participación), se las entiende como instrumentos para la participación y la colaboración entre docentes y estudiantes, que además no tienen que estar situados necesariamente en el mismo espacio y tiempo. Se parte de la perspectiva de que el aprendizaje tiene una dimensión social, y que la formación implica aprender en comunidad y ser capaz de interactuar y colaborar para construir el conocimiento. Es importante tener en cuenta que el aprendizaje ya no se produce solamente en las instituciones educativas, sino que es cada vez más ubicuo; de aquí que la función de las instituciones educativas sea hoy la integración de diferentes aprendizajes que se producen en contextos diferenciados. Supone también que docente y estudiante sean más proactivos, y por tanto no solo consuman información, sino que también la creen.

Desde el Campus virtual de la Universidad Nacional de Lanús, y en consonancia con los citados especialistas, se reconoce la compleja relación entre la adopción de propuestas tecnológicas y las prácticas educativas, por lo que se deben tener en cuenta los recursos (materiales y humanos) así como con la 
formación docente y las iniciativas de políticas educativas. Pero la incorporación de dichas propuestas no es suficiente para mejorar la calidad de la educación; el impacto de la esfera tecnológica en todo el sistema social exige una revisión continua de las condiciones y de los escenarios en los que la mencionada relación se establece. Es importante, entonces, adoptar un pensamiento en prospectiva que permita anticipar los cambios posibles en las instituciones educativas y en sus actores.

\section{Desarrollo del Campus Virtual de la UNLa}

Desde su conformación al cada vez más generalizado uso de las TIC en las prácticas educativas, estas se suman a las políticas ya iniciadas y sostenidas por la universidad, en pos de favorecer la inclusión, la democratización del conocimiento y las mejoras en las propuestas formativas. Se gesta entonces un proceso más amplio de incorporación de dichas herramientas, en el marco de un profundo análisis y reflexión que reconoce la compleja relación entre tecnologías y prácticas universitarias, entendiendo que su mera incorporación no garantiza el cumplimiento del propósito de mejorar los procesos de enseñanza y de aprendizaje.

\subsection{Organización y gestión del Campus Virtual UNLa}

La UNLa crea por la Resolución Rectoral 1589 del año 2009 un Campus virtual, asignado al Vicerrectorado la tarea de su organización (Universidad Nacional de Lanús, 2009). Posteriormente, se crea la dirección de esta dependencia a través de la Resolución del Consejo Superior n. ${ }^{\circ}$ 161/13 (Universidad Nacional de Lanús, 2013b) y en el mismo año por Resolución del Consejo Superior n. ${ }^{\circ} 11 / 13$ se aprueba su Modelo Pedagógico y su Manual de Procedimientos (Universidad Nacional de Lanús, 2013a, p. 10).

El Campus virtual de la UNLa es centralizado y transversal a los departamentos y secretarías, con los que trabaja articuladamente para optimizar las propuestas a distancia de la universidad.

Respecto a los fines del Campus virtual UNLa vale la pena destacar el papel que cumplen las tecnologías digitales en este campo de experiencias, por su capacidad para ampliar las redes de producción y transmisión de conocimiento y por inspirar la creación de ámbitos renovados para el trabajo académico entre docentes $\mathrm{y}$ estudiantes.

En general, las funciones consisten en acompañar a la comunidad educativa en los procesos de acercamiento e inclusión de las tecnologías digitales, en promover espacios de reflexión en torno a la construcción de nuevas identidades digitales, en facilitar el uso de distintas herramientas de comunicación y de socialización de la información y, particularmente, en orientar a todos aquellos docentes que por distintas razones proyectan virtualizar sus espacios curriculares (asignatura, curso, seminario u otro).

Virtualización está definida aquí como el proceso de diseño, planificación, gestión y evaluación de un espacio curricular mediado por tecnologías digitales, lo cual implica, entre otras tareas, la elaboración o selección de materiales didácticos en diversos formatos (escritos, audiovisuales, hipertextuales y otros), la generación de espacios de intercambio académico y demás actividades relacionadas con los procesos de enseñanza y de aprendizaje en el Nivel Superior.

La virtualización implica tomar una serie de decisiones que, a partir del análisis y la evaluación de los contextos de formación, además de las competencias que requiere cada campo profesional, se determina la conveniencia de construir un espacio totalmente virtual o la coexistencia y complementariedad de ambos métodos educativos (presencial y virtual). En la UNLa se pueden reconocer los siguientes usos de su entorno virtual:

- Desarrollo de ofertas de educación a distancia.

- Apoyo a la enseñanza presencial con "aulas extendidas".

La modalidad de enseñanza presencial aprovecha las posibilidades que le brinda el entorno virtual para enriquecer la propuesta pedagógica. Este es el caso del Ciclo de Licenciatura en Economía empresarial, que incluye en su plan de estudio el uso de aulas extendidas para todas las materias del plan.

En la actualidad, el Campus virtual UNLa está conformado por docentes especializados y organizados en diferentes equipos de intervención: Asesoramiento Didáctico, Diseño y Comunicación, Capacitación Pedagógica y Tecnológica, Desarrollo e Innovación, y Administración Técnica.

El equipo de Asesoramiento Didáctico brinda asesoramiento para la planificación, gestión y evaluación del espacio curricular. El equipo de Diseño y Comunicación realiza el diseño de los materiales de estudio 
y del entorno visual de las propuestas curriculares virtuales, así como el diseño de las comunicaciones internas y externas del Campus virtual UNLa. El equipo de Capacitación Pedagógica y Tecnológica realiza capacitaciones grupales e individuales relacionadas con el uso de la plataforma educativa de la universidad y a las características de las aulas virtuales. El equipo de Desarrollo e Innovación desarrolla y actualiza propuestas informáticas asociadas al entorno virtual de enseñanza y de aprendizaje, y también desarrolla, adapta y actualiza los sistemas de comunicación, con el objetivo de optimizar la gestión de dicho entorno y de difundir las actividades y propuestas de las diferentes áreas que lo integran. Por último, el equipo de Administración Técnica se encarga de realizar las tareas de mantenimiento de la plataforma tecnológica del Campus virtual UNLa y de brindar soporte técnico y administrativo a los usuarios.

Además de estos equipos debe mencionarse la tarea que lleva a cabo el Equipo de Referentes del Campus virtual, que está integrado por docentes o personal administrativo perteneciente a departamentos o secretarías de la universidad. Cada uno de ellos es elegido por los directores de esas dependencias para desempeñar el rol de nexo entre las carreras o cursos y el Campus virtual, con el objetivo de organizar y centralizar las inquietudes y necesidades de los directores, docentes, asistentes y estudiantes con respecto al uso del entorno virtual en la institución en el contexto de una propuesta de educación a distancia.

\section{Modelo pedagógico del Campus Virtual UNLa}

Desde sus inicios, nuestra universidad ha reflexionado sistemáticamente sobre el modelo institucional y su inserción en un contexto de cambio. Como expresa Jaramillo (2008):

La información se genera, se acumula y distribuye cada vez más por el ciberespacio. La economía súper simbólica y la tecnología ciberespacial derrumban día a día no solo las paredes áulicas de los centros de enseñanza sino de las fronteras nacionales (...). Una sociedad del conocimiento como la que estamos viviendo, requiere modificar nuestro concepto de universidad claustro por el de universidad-ciudad del conocimiento. Solo así, la universidad podrá aspirar a desempeñar su función protagónica en la creación de una nueva civilización y un nuevo futuro (p. 58).

Por esta razón, la universidad continúa atendiendo a las transformaciones propiciadas por la innovación tecnológica y por el desarrollo de las más recientes TIC que hoy son medios poderosos para la innovación de las propuestas de formación universitaria.

El Modelo Pedagógico que se propone responde, como ya se anticipó, a los lineamientos del Proyecto Institucional de la UNLa, creada a las puertas del siglo XXI, "cuando el conocimiento se ha convertido en la fuerza productiva mayor de la humanidad" (Universidad Nacional de Lanús, 1998, p. 48). Dicho proyecto promueve la construcción de una ciudad del conocimiento y conlleva la función de articular a los productores de saberes científicos, socioculturales, tecnológicos y políticos.

La UNLa, al configurar su sistema institucional de educación a distancia, propone un modelo pedagógico afín a estas ideas, destacando que la articulación interdisciplinaria, la relación de la universidad con los problemas de la sociedad y la intervención en la práctica (como estrategias didácticas y apoyadas en entornos digitales) potencian la construcción de conocimientos, el trabajo en red y enriquecen la comunicación entre todos los actores involucrados.

\section{Potencialidades con que se cuenta y obstáculos y dificultades que se prevén}

En la universidad existen potencialidades institucionales que fortalecen las propuestas en la modalidad a distancia. Entre ellas pueden destacarse las decisiones de innovación y desarrollo del parque tecnológico, la incorporación de profesionales de distintos campos disciplinarios, la permanente ampliación de la oferta académica y, especialmente, los procesos de reflexión compartida e interdisciplinaria que van acompañando las transformaciones de orden tecnológico, comunicacional y pedagógico. Esto permite que el Campus virtual sea transversal a los departamentos académicos, que se observan tanto en la reconceptualización de los espacios de intervención inicialmente previstos como en sus producciones.

Con respecto a las dificultades a superar, cabe ampliar y fortalecer un conjunto de acciones que convoquen a mayor número de docentes a la participación en experiencias educativas con inclusión tecnológica, pues con ellas se abre la oportunidad de redefinir los modos de operación con el saber que se privilegian en la cultura universitaria. Este problema no es privativo de UNLa, dado que, en general, atraviesa las prácticas 
docentes de los distintos niveles del sistema educativo. Esto ha sido explicado un tanto "livianamente", en términos de "resistencia" y "conservadurismo" (Dussel et al., 2010, pp. 85-89).

Lo interesante de este enfoque es que abre una serie de debates que todavía no se han arraigado en las propuestas de formación de los docentes, al menos de forma masiva, a pesar de comprobar por medio de algunas encuestas e investigaciones, el apoyo de los profesores a los medios digitales en las aulas. Pero coincidiendo con el planteamiento de Dussel et al., (2010) puede agregarse que, pese a ser usuarios frecuentes de las tecnologías, buena parte de los docentes poseen ciertas limitaciones para la planificación de un nuevo repertorio de estrategias didácticas con dichos recursos.

Todas estas son cuestiones sobre las que la institución debe seguir trabajando en una articulación intrainstitucional, privilegiando el diálogo al interior del colectivo docente.

\section{Conclusiones}

Hasta aquí se han señalado de manera general aquellos aspectos e intencionalidades que fundamentan la construcción de un Proyecto Institucional de Educación a Distancia en la Universidad Nacional de Lanús.

A modo de síntesis se destaca el valor que tiene esta modalidad como opción pedagógica que, enriquecida por la inclusión de tecnologías digitales, favorece la producción y circulación de saberes en contextos cada vez más amplios de interacción social, y en este sentido, se articula con los principios del marco institucional UNLa antes mencionados, que resaltan el carácter público del conocimiento y la democratización para el acceso al mismo.

La inclusión de las TIC permite generar procesos de reflexión en torno al sentido, el significado y las maneras de diseñar propuestas educativas capaces de enriquecer el marco actual de las prácticas universitarias. Esto significa que, si bien la reflexión es inherente a la enseñanza entendida como proceso intencional ciertamente guiado, metódico, pero a la vez flexible para considerar los cambios que mejor se ajusten a distintos contextos y grupos (Davini, 2015), también es posible afirmar que la inclusión de las tecnologías en las experiencias educativas abre oportunidades para que los docentes puedan interpelar su propio quehacer y el de otros, en dirección a transformar las prácticas de enseñanza de la universidad.

Por último, existe la necesidad de hacer una constante evaluación de la incorporación de las TIC a la enseñanza universitaria (específicamente en la UNLa) con la finalidad de producir conocimiento que permita brindar elementos para la toma de decisiones, transferir los aprendizajes surgidos de experiencias innovadoras y orientar el mejoramiento de la calidad y de la democratización de la educación superior (Neirotti, 2005, p. 97).

Todo esto con la intención de que este proceso de evaluación sea un aprendizaje y que constituya un momento reflexivo de la acción, que ayude a ordenar la intervención y la gestión, asumiendo que dicha evaluación ayude a tomar mayor conciencia de las limitaciones y a desarrollar la capacidad de solucionar las limitaciones de las que se toma conciencia (Neirotti, 2005, p. 98).

\section{Agradecimientos}

Este proyecto institucional se desarrolla gracias al acompañamiento de un equipo de trabajo interdisciplinario que de manera articulada realiza actividades de enseñanza, investigación y cooperación. Gracias a este equipo es posible pensar en una gestión de calidad e inclusiva, por lo que acá se le hace un reconocimiento especial. Pueden consultarse las actividades desarrolladas por el Campus virtual UNLa en el siguiente enlace: http://campus.unla.edu.ar/

\section{Declaración de conflicto de intereses}

Las autoras declaran no tener conflicto de intereses con respecto a la investigación, autoría o publicación de este artículo.

\section{Referencias}

Cabero Almenara, J. (2015). Reflexiones educativas sobre las tecnologías de la información y comunicación. Tecnología, Ciencia y Educación, 1, 19-27.

Davini, M. C. (2015). La formación en la práctica docente. Colección voces de la educación. Ed. Paidós, Buenos Aires, Argentina. 
Dussel, I., Minzi, V., Mosenson, F., Pagola, L., y Peirone, F. (2010). La educación alterada. Aproximación a la escuela del siglo XXI. Salida al Mar Ediciones.

Fainholc, B. (1999). La interactividad en la educación a distancia. Ed. Paidós, Buenos Aires, Argentina.

García Aretio, L., Ruiz Corbella, M., y Domínguez Figaredo, D. (2007). De la educación a distancia a la educación virtual. Editorial Ariel, Barcelona, España.

Jaramillo, A. (2008). Universidad y proyecto nacional. Remedios de Escalada. Ediciones de la UNLa Universidad Nacional de Lanús, Buenos Aires, Argentina.

Neirotti, N. (2005). Elementos conceptuales y metodológicos para la evaluación de políticas y programas sociales. Buenos Aires, Argentina. Recuperado de https://campus.fundec.org.ar/admin/archivos/Neirotti 2005 Elementos conceptuales y metodológicos.pdf

Rifkin, J. (2014). La sociedad de coste marginal cero: el internet de las cosas, el procomún colaborativo y el eclipse del capitalismo. Ediciones Paidós Ibérica, Barcelona, España.

Universidad Nacional de Lanús. (1998). Proyecto institucional de la UNLa.

Universidad Nacional de Lanús. (2009). Resolución Rectoral No 1589/09.

Universidad Nacional de Lanús. (2013a). Resolución del Consejo Superior No 11/13.

Universidad Nacional de Lanús. (2013b). Resolución del Consejo Superior No 161/13.

Universidad Nacional de Lanús. (2014). Estatuto de la Universidad Nacional de Lanús. Recuperado de http://www. unla.edu.ar/documentos/resoluciones_asamblea/R.AU.N 001-14--30.01.14 Resolucion modificacion Estatuto.pdf

\section{Sobre los autores}

\section{Laura Virginia Garbarini}

Profesora de Historia egresada de la Facultad de Filosofía y Letras de la Universidad de Buenos Aires (UBA) y doctoranda en Educación en la Universidad Nacional de Lanús (UNLa). Profesora investigadora en el Departamento de Planificación y Políticas Públicas de la Universidad Nacional de Lanús, desde 1997. Consejera departamental por el claustro docente para el período 2014-2017 y 2017-2020. Investigadora en el tema de educación virtual y políticas públicas de educación con tecnologías.

\section{María Sol Quiroga}

Arquitecta graduada en la Facultad de Arquitectura y Urbanismo (FAU), Universidad Nacional de La Plata, (UNLP), Especialista en Historia y Crítica de la Arquitectura y del Urbanismo por la Facultad de Arquitectura, Diseño y Urbanismo (FADU), Universidad Nacional de Buenos Aires (UBA), y Doctora en Ingeniería de Proyectos en Medio Ambiente, Seguridad, Calidad y Comunicación, por la Escola Tècnica Superior d' Enginyeria Industrial (ETSEIB), Universitat Politècnica de Catalunya (UPC), Barcelona, España. 